\title{
Risks and forms of cancer associated with alcohol consumption
}

\author{
Rodolfo do Couto Maia \\ Department of Chemistry, University College London, 20 Gordon Street, London, United Kingdom
}

Email address:

rodolfomia10@yahoo.com.br (R. do C. Maia)

\section{To cite this article:}

Rodolfo do Couto Maia. Risks and Forms of Cancer Associated with Alcohol Consumption. Cancer Research Journal. Special Issue: Lifestyle and Cancer Risk. Vol. 2, No. 6-1, 2014, pp. 30-33. doi: 10.11648/j.crj.s.2014020601.13

\begin{abstract}
Although alcohol has been considered a carcinogenic agent for a long time, there still was a lot of controversy about many questions on the role of alcohol in several types of cancer. During the last few years some studies have been trying to address these many issues and the understanding about this topic has greatly increased. Some of these data have been gathered and highlighted in this review as a way to demonstrate if and/or how alcohol consumption, in combination or not with other unsafe lifestyle habits, in fact represents a risk factor for some cancer types that it has been associated with.
\end{abstract}

Keywords: Cancer, Alcohol, Alcohol Consumption, Cancer Risk, Lifestyle

\section{Introduction}

Alcohol consumption is one of the oldest and more prevalent lifestyle social habits of the contemporary society, being a significant part of the lives of billions of people worldwide. Unfortunately, it has been very well-established as a risk factor in the development of several types of cancer, where $3.6 \%$ of all cancers (5.2\% in men, $1.7 \%$ in women) are attributable to alcohol drinking [1 - 3]. However, this issue is often neglected because it is hard to be adequately addressed and discussed due to its complex and heterogeneous nature that comes from all the demographic and socioeconomic diversity in alcohol consumption as well the well-established gender differences (i.e., consistently higher consumption in males than in females) [4].

In addition to all of this, alcohol consumption usually is accompanied by other cancer risk factors, such as bad lifestyle and dietary habits, which makes even more difficult to measure the proper weight that alcohol consumption actually has in the cancer development [1]. Also, it still should be considered that there are several types of alcoholic beverages (i.e., beer, wine, spirits), amount of alcohol drinking (i.e., light, moderate or high intake), diverse patterns of consumption (i.e., weekend heavy drinkers, everyday light drinkers, etc). Although there is no consensus on the 'safe' level of alcohol consumption in general population, it is conceivable to think that each type of alcohol-associated cancer is more sensible to one of the aforementioned scenarios or to a combination of them $[5,6]$.
In this context, the aim of this review is to present the types of cancer that have been more associated with alcohol consumption and highlight studies (mainly more recent studies) where the controversial aspects about the alcohol intake association with cancer risk was approached. The highlights will include which kind of lifestyle profile related to alcohol consumption (e.g., frequency/range of alcohol drinking, tobacco association or not) starts to threaten an increase in risk of cancer and even if there is a risk at all.

\section{Alcohol-Related Types of Cancer and Consumption-Associated Risks}

\subsection{Breast Cancer}

First, among women, breast cancer is the most common cancer worldwide and the leading cause of cancer-related mortality. Consumption of alcoholic beverages is causally associated with female breast cancer. The role of heavy drinking has been long recognized and even a moderate intake is associated with an increased risk for breast cancer [7]. The relationship between alcohol consumption and breast cancer risk is shown by epidemiologic studies, and corroborated by animal and in vitro evidence [8]. Most studies reported a linear dose-response with increasing daily or cumulative intake [7, 9, 10].

Cumulative lifetime consumption, drinking frequency, drinking patterns and timing of exposure each modulate the association between alcohol consumption and breast cancer. 
Hormonal status, genetic polymorphisms, and nutritional factors may interact with ethanol metabolism and further influence breast cancer risk [7, 11]. In general, the difference in breast cancer risk between different types of alcoholic beverage is not consistent and provides further evidence that ethanol is the main agent $[12,13]$.

Interestingly, a study demonstrated that pre- and postoperative alcohol consumption was not related to risk for early breast cancer events, stating that recommending that all breast cancer patients abstain from low to moderate alcohol consumption could not be supported [14]. Another study indicates that light drinking during early adulthood may be beneficial, but heavy drinking may be related to increased risk of mortality among women with breast cancer [15].

Looking at the general results from all the studies, it is clear that even light consistent consumption of alcohol can promote breast cancer in the long term.

\subsection{Liver Cancer}

Alcohol has been associated with increased risk of primary liver cancer, however, the major risk factors for hepatocellular carcinoma (HCC), the most frequent histological type of primary liver cancer, is not alcohol but persistent infection with hepatitis $\mathrm{B}$ virus (HBV) and hepatitis $\mathrm{C}$ virus (HCV), although alcohol may enhance and/or accelerate hepatocarcinogenesis in patients with $\mathrm{HBV}$ and/or $\mathrm{HCV}$ infection [16, 17]. A recent study has shown that the consumption of more than 3 alcoholic drinks per day increased the liver cancer risk, compared with no alcohol consumption [18].

\subsection{Oral Cancer}

Before Since the 70's, it is well known that alcohol consumption can contribute to oral cancer development, especially when combine with tobacco smoking [17, 19]. However, it was recently shown that the independent effects of smoking and drinking on oral cancer risk are lower than expected. In fact, it was demonstrated that the independent effect of drinking substantially decreased and was no longer associated with oral and oropharyngeal cancer when compared to the smoking-drinking combination [20]. According to these findings, oral cancer control policies should focus primarily on the addictive behavior that induces people to adopt several unsafe lifestyles.

\subsection{Prostate Cancer}

Although alcohol has not been established as risk factor for prostate cancer, it is an important risk factor for other human cancers. Noteworthy is a recent study of 50 case-control studies and 22 cohort that concluded that there was no evidence of a material association between alcohol drinking and prostate cancer [21]. On the other hand, another study found that alcohol drinking increases the risk of advanced prostate cancer. They further suggest that abstinence from alcohol and prohibition of smoking might be an important factor in the prevention of advanced prostate cancer [22].
Another study made the correlation between alcohol consumption and PSA-detected prostate cancer risk. Their results support the hypothesis that heavy alcohol drinking causes a small increase in risk of high-grade prostate cancer, and are general to European-origin populations with widespread use of PSA testing. However, no firm conclusion could be reached on the nature of the effect of alcohol on low-grade cancers [23].

Finally, there is a study regarding the lifetime alcohol intake and the prostate cancer risk. The results indicated that current alcohol intake did not increase prostate cancer risk but lifetime intake increased risk for both nonaggressive and aggressive cases, supporting the evidence for an increased risk of prostate cancer from lifetime alcohol consumption [24].

\subsection{Upper Aerodigestive Tract Cancer}

Upper aerodigestive tract (UADT) cancer, including the cancer of oral cavity, pharynx, larynx, and esophagus, is among the most frequent cancer and the most common death causes of cancer in the world [25]. As for oral cancer, the combination of alcohol consumption and tobacco smoking is far more dangerous than the abuse of either one of them alone $[17,19]$. Until some point, there was still substantial uncertainty if any potential relation between alcohol intake and UADT cancer mortality is true or just due to residual confounding by smoking or other factors. A recent publication provides evidence of a positive association between alcohol drinking and UADT cancer mortality, especially at moderate-to-heavy level [26]. Another study demonstrated that alcohol drinking in patients with UADT cancer is associated with an increased risk of second primary cancers [27]. Thus, public health recommendations on UADT cancer prevention and control should consider limiting the intake of alcoholic beverages.

One major hypothesized mechanism behind alcohol-related carcinogenesis in the UADT is the involvement of acetaldehyde, the main metabolite of ethanol. Aldehyde dehydrogenase 2 (ALDH2) is a key enzyme in acetaldehyde metabolism, and molecular epidemiologic studies in East Asia [28-30], where the functional $A L D H 2$ Glu504Lys (rs671) polymorphism is prevalent, have contributed to the conclusion that acetaldehyde has a substantial impact on carcinogenesis in humans as a result of its strong interaction with alcohol drinking [3].

\subsection{Stomach Cancer}

The impact of alcohol on the risk of stomach cancer is controversial. To date, there is no strong evidence associating alcohol consumption with stomach cancer, although it has been hypothesized that acetaldehyde contributes to gastric carcinogenesis, as it does for UADT cancer [29, 30]. A recent study investigated the association between ALDH2 Glu504Lys (rs671) polymorphism and alcohol intake with the risk of stomach cancer in Japanese population. It was found that ALDH2 and alcohol drinking interact with each other in the risk of stomach cancer. This result indicates an important 
role of acetaldehyde in the stomach cancer development, as has already been shown for cancers of the UADT [31].

\subsection{Endometrial Cancer}

Alcohol consumption may increase the risk of endometrial cancer by adversely affecting the concentrations of steroids in both pre- and postmenopausal women [32, 33]. However, a moderate alcohol intake has been shown to have a beneficial effect on endometrial cancer risk [34, 35]. Trying to address this issue, Ferdiko et al performed a large, multicenter, prospective study to understand the association between alcohol intake and endometrial cancer risk. They found that alcohol drinking has no association with endometrial cancer risk [36].

\subsection{Skin Cancer}

Alcohol Skin cancer is one of the most common malignancy, if not the most common. A dramatic increase in the incidence of both malignant melanoma (MM) and non-melanoma skin cancer (NMSC) has been observed during the past decades, especially among women. It is well known that sun exposure and host factors such as skin color and lifestyle factors have been shown to play a crucial role in the development of skin cancers $[37,38]$. Therefore, as alcohol drinking is a key risk factor for many malignancies, it is reasonable to consider that both amount and type of alcohol consumed may potentially be a risk factor for skin cancers. A study with 59,575 white postmenopausal women, median age of 63.6, was performed and the results have indicated that a higher current alcohol consumption, higher lifetime alcohol consumption, and a preference for white wine or liquor were associated with increased hazard of MM and risk of NMSC [39].

\section{Conclusions}

The recent data published in the literature leaves no doubt about the carcinogenic potential of alcohol consumption for several types of cancer. Although, it is interesting to note the wildly varying range of consumption patterns associated with each type of cancer that alcohol drinking presents a risk. While some forms of cancer are associated only with a lifetime alcohol intake, even if a light one, to actually present a cancer risk, others are related to any amount of intake at any time of one's life. We also have cases where the actual danger is the combination of bad lifestyle habits, such as smoking, drinking and lack of exercises, where they act synergistically, but separated they pose much less or none cancer risk. This leads to an interesting conclusion that, for some types of cancer, it is actually better to have only one intense bad lifestyle component, e.g. heavy alcohol drinking, than the presence of three light bad lifestyle factors, e.g. light smoking, light drinking and bad dietary habits. This is an important finding because cancer control policies should focus primarily on the addictive behavior that induces people to adopt several unsafe lifestyles and knowing that each one that people can successfully quit will in fact generate great gains towards the reduction of cancer incidence. Finally, many of the controversy around the role of alcohol as a cancer risk factor has been elucidated in the last few years and there is a better understanding of the synergies between several others lifestyle risk factors.

\section{References}

[1] M. Touvier, N. Druesne-Pecollo, E. Kesse-Guyot, V. A. Andreeva, P. Galan, S. Hercberg, and P. Latino-Martel, "Demographic, socioeconomic, disease history, dietary and lifestyle cancer risk factors associated with alcohol consumption” Int. J. Cancer, vol. 134, pp. 445-459, 2014.

[2] American Institute for Cancer Research/World Cancer Research Fund. Food, nutrition, physical activity and the prevention of cancer: a global perspective. Washington, DC: American Institute for cancer research, 2007.

[3] IARC. IARC Monographs on the Evaluation of Carcinogenic Risks to Humans Volume 100E. A review of human carcinogens: personal habits and indoor combustions. Consumption of alcoholic beverages. 2012; 373-499.

[4] P. Dias, A. Oliveira, and C. Lopes, "Social and behavioural determinants of alcohol consumption" Ann. Hum. Biol, Vol. 38, pp. 337-344, 2011.

[5] V. Bagnardi, et al. "Light alcohol drinking and cancer: a meta-analysis” Ann. Oncology, vol. 24, pp. 301-308, 2013.

[6] M. Jin, et al. "Alcohol drinking and all cancer mortality: a meta-analysis" Ann. Oncology, vol. 24, pp. 807-816, 2013.

[7] C. Scoccianti, B. Lauby-Secretan, P.-Y. Bello, V. Chajes, I. Romieu, "Female Breast Cancer and Alcohol Consumption" Am. J. Prev. Med, vol. 46, pp. S16-S25, 2014.

[8] B. Secretan, et al. "A review of human carcinogens-- Part E: tobacco, areca nut, alcohol, coal smoke, and salted fish" Lancet Oncol, vol. 10, pp.1033-4, 2009.

[9] S. M. Zhang, I. M. Lee, J. E. Manson, N. R. Cook, W. C. Willett, and J. E. Buring, "Alcohol consumption and breast cancer risk in the Women's Health Study, " Am. J. Epidemiol, vol. 165, pp. 667-76, 2007.

[10] Y. Li, D. Baer, G. D. Friedman, N. Udaltsova, V. Shim. and A. L. Klatsky, "Wine, liquor, beer and risk of breast cancer in a large population" Eur. J. Cancer, vol. 45, pp. 843-50, 2009.

[11] P. J. Brooks and S. Zakhari, "Moderate alcohol consumption and breast cancer in women: from epidemiology to mechanisms and interventions" Alcohol Clin. Exp. Res, vol. 37, pp. 23-30, 2013.

[12] P. A.Newcomb, et al. "No difference between red wine or white wine consumption and breast cancer risk" Cancer Epidemiol. Biomarkers. Prev, vol. 18, pp.1007-10, 2009.

[13] N. E. Allen, et al. "Moderate alcohol intake and cancer incidence in women" J. Natl. Cancer Inst, vol. 101, pp. 296-305, 2009.

[14] M. Simonsson, A. Markkula, P.-O. Bendahl, C. Rose, C. Ingvar, and H. Jernström, "Pre- and postoperative alcohol consumption in breast cancer patients: impact on early events" SpringerPlus, vol. 3, pp. 261, 2014. 
[15] A. M. Weaver, S. E. McCann, J. Nie, S. B. Edge, T. H. Nochajski, M. Russell, M. Trevisan, and J. L. Freudenheim, "Alcohol intake over the life course and breast cancer survival in Western New York exposures and breast cancer (WEB) study: quantity and intensity of intake" Breast Cancer Res. Treat, vol. 139, pp. 245-253, 2013.

[16] H. K. Seitz and F. Stickel. "Risk factors and mechanisms of hepatocarcinogenesis with special emphasis on alcohol and oxidative stress” Biol. Chem, vol. 387, pp. 349-360, 2006.

[17] IARC. IARC Monographs on the evaluation of carcinogenic risks to humans. vol. 96. Alcohol Consumption and Ethyl Carbamate. Lyon: International Agency for Research on Cancer, 2010.

[18] F. Turati, C. Galeone, M. Rota, C. Pelucchi, E. Negri, V. Bagnardi, G. Corrao, P. Boffetta, and C. La Vecchia, "Alcohol and liver cancer: a systematic review and meta-analysis of prospective studies" Ann. Oncology, vol. 25, pp. 1526-1535, 2014.

[19] S. Petti, "Lifestyle risk factors for oral cancer" Oral. Oncol, vol. 45, pp. 340-350, 2009.

[20] J. L. F. Antunes, T. N. Toporcov, M. G. H. Biazevic, A. F. Boing, C. Scully, and S. Petti, "Joint and Independent Effects of Alcohol Drinking and Tobacco Smoking on Oral Cancer: A Large Case-Control Study" PLOS ONE, vol 8, pp.e68132, 2013.

[21] M. Rota, et al. "Alcohol consumption and prostate cancer risk: a meta-analysis of the dose-risk relation" Eur. J. Cancer Prev, vol. 21, pp. 350-9, 2012.

[22] N. Sawada, M. Inoue, M. Iwasaki, S. Sasazuki, T. Yamaji, T. Shimazu, and S. Tsugane, "Alcohol and smoking and subsequent risk of prostate cancer in Japanese men: The Japan Public Health Center-based prospective study" Int. J. Cancer, vol. 134, pp. 971-978, 2014.

[23] L. Zuccolo, S. J. Lewis, J. L. Donovan, F. C. Hamdy, D. E. Neal, and G. D. Smith, "Alcohol consumption and PSA-detected prostate cancer risk-A case-control nested in the ProtecT study" Int. J. Cancer, vol. 132, pp. 2176-2185, 2013.

[24] S. E. McGregor, K. S. Courneya, K. A. Kopciuk, C. Tosevski, and C. M. Friedenreich, "Case-control study of lifetime alcohol intake and prostate cancer risk" Cancer Causes Control, vol. 24, pp. 451-461, 2013.

[25] J. Ferlay, H. R. Shin, F. Bray, D. Forman, C. Mathers, and D. M Parkin, "Estimates of worldwide burden of cancer in 2008: GLOBOCAN 2008” Int. J. Cancer, vol.127, pp. 2893-917, 2010 .

[26] Y. Li, et al. "Alcohol drinking and upper aerodigestive tract cancer mortality: A systematic review and meta-analysis" Oral Oncology, vol. 50, pp. 269-275, 2014.

[27] N. Druesne-Pecollo, et al. , "Alcohol Drinking and Second Primary Cancer Risk in Patients with Upper Aerodigestive
Tract Cancers: A Systematic Review and Meta-analysis of Observational Studies, " Cancer Epidemiol. Biomarkers Prev, vol. 23, pp. 324-331, February, 2014.

[28] K. Matsuo, et al. "Gene-environment interaction between an aldehyde dehydrogenase-2 (ALDH2) polymorphism and alcohol consumption for the risk of esophageal cancer" Carcinogenesis, vol. 22, pp. 913-916, 2001.

[29] M. Salaspuro, "Acetaldehyde as a common denominator and cumulative carcinogen in digestive tract cancers" Scand. J. Gastroenterol, vol. 44, pp. 912-925, 2009.

[30] M. Salaspuro, “Acetaldehyde and gastric cancer" J. Dig. Dis, vol. 12, pp. 51-59, 2011.

[31] K. Matsuo, I. Oze, S. Hosono, H. Ito, M. Watanabe, K. Ishioka, S. Ito, M. Tajika, Y. Yatabe, Y. Niwa, K. Yamao, S. Nakamura, K. Tajima, and H. Tanaka, "The aldehyde dehydrogenase 2 (ALDH2) Glu504Lys polymorphism interacts with alcohol drinking in the risk of stomach cancer" Carcinogenesis, vol.34, pp.1510-1515, 2013.

[32] S. Rinaldi , P. H. Peeters, I. D. Bezemer, et al, "Relationship of alcohol intake and sex steroid concentrations in blood in preand post-menopausal women: the European Prospective Investigation into Cancer and Nutrition" Cancer Causes Control, vol. 17, pp.1033-43, 2006.

[33] A. M. McDivit, G. A. Greendale, F. Z. Stanczyk, and M. H. Huang, "Effects of alcohol and cigarette smoking on change in serum estrone levels in postmenopausal women randomly assigned to fixed doses of conjugated equine estrogens with or without a progestin" Menopause, vol. 15, pp. 382-5, 2008.

[34] A. Imhof, M. Froehlich, H. Brenner, H. Boeing, M. B. Pepys, and W. Koenig, "Effect of alcohol consumption on systemic markers of inflammation" Lancet, vol. 357, pp. 763-7, 2001.

[35] J. K. Pai, S. E. Hankinson, R. Thadhani, N. Rifai, T. Pischon, and E. B. Rimm, "Moderate alcohol consumption and lower levels of inflammatory markers in US men and women" Atherosclerosis, vol. 186, pp. 113-20, 2006.

[36] V. Fedirko, et al. "Alcohol drinking and endometrial cancer risk in the European Prospective Investigation into Cancer and Nutrition (EPIC) study" Ann. Epidemiol, vol. 23, pp. 93-98, 2013.

[37] E. Linos, S. M. Swetter, M. G. Cockburn, G. A. Colditz, and C. A. Clarke, "Increasing burden of melanoma in the United States” J. Invest. Dermatol, vol. 129, pp. 1666-1674, 2009.

[38] H. W. Rogers, et al. "Incidence estimate of nonmelanoma skin cancer in the United States" Arch. Dermatol, vol. 146, pp.283-287, 2010.

[39] J. T. Kubo, M. T. Henderson, M. Desai, J. Wactawski-Wende, M. L. Stefanick, and J. Y. Tang, "Alcohol consumption and risk of melanoma and non-melanoma skin cancer in the Women's Health Initiative" Cancer Causes Control, vol. 25, pp. 1-10, 2014. 